$$
B U-1068-M
$$

\title{
PAIR FORMATION IN STRUCTURED POPULATIONS
}

Carlos Castillo-Chavez

Biometrics Unit/Center for Applied Math.

341 Warren Hall

Cornell University

Ithaca, NY 14853-7801

U.S.A.

$\begin{array}{ll}\text { Stavros Busenberg } & \text { Ken Gerow } \\ \text { Department of Mathematics } & \text { Biometrics Unit } \\ \text { Harvey Mudd College } & 337 \text { Warren Hall } \\ \text { Claremont, California 91711 } & \text { Cornell University } \\ \text { U.S.A. } & \text { Ithaca, NY 14853-7801 } \\ & \text { U.S.A. }\end{array}$

Abstract

Pair formation or social mixing has become one of the central problems in the study of the dynamics of sexually-transmitted diseases. In this paper we outline a unified approach to pair formation for one- and two-sex populations by means of an axiomatic mixing framework. We also illustrate numerically the effects of the structural covariance or preference function (a measure of deviation from proportionate mixing) on the mixing or pair formation function for homosexual populations. In addition, a two-sex demographic model that follows pairs is formulated and briefly analyzed.

1. Introduction

The grim scenario created by the AIDS epidemic has driven researchers to develop mathematical models to improve our understanding of the mechanisms responsible for HIV (the etiological agent for AIDS) transmission and of the evaluation of possible intervention measures. Recent reviews of the literature on models include those of Anderson (1988, 1989), Castillo-Chavez (1989a,b), and Schwager et al. (1989). Some of the important conclusions generated by mathematical models include the clear 
identification of three key mechanisms: variable infectivity, mixing or pair formation, and long, variable periods of infectiousness, which have the greatest effect on HIV transmission at the population level. For an extensive in depth study of some of the most recent mathematical and statistical work in these and other areas related to AIDS epidemiology see Castillo-Chavez (1989b).

This paper is organized as follows: in Section 2, we outline a unified axiomatic approach to the problem of mixing which extends and generalizes the one-sex framework of Blythe and Castillo-Chavez (1989) and ,Castillo-Chavez and Blythe (1989) and provide an expression for the general solution as well as some numerical illustrations; in Section 3, we formulate a two-sex mixing or pair formation framework that is a natural generalization of the one-sex framework, and construct some explicit solutions; in Section 4 we formulate a demographic model that follows pairs and provides some preliminary analysis.

\section{Mixing framework}

The formulation described in this section can be used in the modeling of social or sexual mixing interactions. The mixing or pair formation function can describe the proportion of "dates" between individuals in distinct groups, or it can represent the proportion of sexual partnerships or sexual contacts between these individuals. In addition, the mixing function can be generalized to include the geographical distribution or the geographical movement of individuals through the use of "localized"

mixing functions, i.e., functions that represent the proportion of partnerships formed between individuals from clearly defined groups (social, demographic, etc.) at a particular geographical location. The local geographical heterogeneities can then be linked through the specification of migration or movement matrices (see Sattenspiel 1987a, b, Sattenspiel and Simon 1988). Therefore, our approach allows for the specification of a spatial mixing framework. In this paper, however, we concentrate in the study of localized mixing functions.

Since our work has been motivated by HIV dynamics, we concentrate on the study of mixing functions in the context of SIR models where $S$ represents the class of susceptible individuals, I the class of infected individuals, and $R$ the class of removed or recovered individuals. We consider first the interactions of a single, socially-homogeneous group of individuals who are structured according to the following variables: $\mathrm{a}=$ age; $\tau=$ time (or age) since infection; $\mathrm{r}=$ activity or risk level. We let $\mathrm{N}(\mathrm{r}, \mathrm{a}, \tau, \mathrm{t})$ denote the total population density per unit age, activity, and time since infection, at time t. This population is divided into the following epidemiological classes: $\mathrm{S}=$ susceptible; $\mathrm{I}=$ 
asymptomatic or slightly symptomatic infective; $\mathrm{A}=$ highly symptomatic infective. This classification is fairly general and includes implicitly the traditional exposed, but not infected, class E (see Busenberg and Castillo-Chavez 1989). In our discussion, $\tau$ is a hidden internal variable that does not distinguish individuals other than through their level of infectivity, and perhaps mortality. When modeling the sexual transmission of AIDS, we assume that A-individuals (i.e. individuals with severe symptoms or "full-blown" AIDS) are sexually inactive (i.e. this class represents the "removed" individuals) and hence that

$$
\mathrm{T}(\mathrm{r}, \mathrm{a}, \mathrm{t})=\mathrm{S}(\mathrm{r}, \mathrm{a}, \mathrm{t})+\int_{0}^{\infty} \mathrm{I}(\mathrm{r}, \mathrm{a}, \tau, \mathrm{t}) \mathrm{d} \tau
$$

represents the total age and activity-level density of a population active in disease transmission contacts. Sexual mixing (or pair formation) is defined through the mixing function $\rho$. Specifically,

$$
\begin{aligned}
\rho\left(r, a, r^{\prime}, a^{\prime}, t\right)= & \text { the proportion of partners of an }(r, a) \text { individual } \\
& \text { (i.e., a person of activity level } r \text { at age a), with }\left(r^{\prime}, a^{\prime}\right) \\
& \text { individuals at time } t . \\
\mathrm{C}(r, a, t)= & \text { the expected or average number of partners per unit time } \\
& \text { of an }(r, a) \text { individual given at time t. We assume } C \geq 0 .
\end{aligned}
$$

The following natural conditions characterize the mixing function:

(i) $\rho \geq 0$,

(ii) $\int_{0}^{\infty} \int_{0}^{\infty} \rho\left(r, a, r^{\prime}, a^{\prime}, t\right) \mathrm{dr}^{\prime} \mathrm{da}^{\prime}=1$,

(iii) $\rho\left(\mathrm{r}, \mathrm{a}, \mathrm{r}^{\prime}, \mathrm{a}^{\prime}, \mathrm{t}\right) \mathrm{C}(\mathrm{r}, \mathrm{a}, \mathrm{t}) \mathrm{T}(\mathrm{r}, \mathrm{a}, \mathrm{t})=\rho\left(\mathrm{r}^{\prime}, \mathrm{a}^{\prime}, \mathrm{r}, \mathrm{a}, \mathrm{t}\right) \mathrm{C}\left(\mathrm{r}^{\prime}, \mathrm{a}^{\prime}, \mathrm{t}\right) \mathrm{T}\left(\mathrm{r}^{\prime}, \mathrm{a}^{\prime}, \mathrm{t}\right)$,

(iv) $\mathrm{C}(\mathrm{r}, \mathrm{a}, \mathrm{t}) \mathrm{T}(\mathrm{r}, \mathrm{a}, \mathrm{t}) \mathrm{C}\left(\mathrm{r}^{\prime}, \mathrm{a}^{\prime}, \mathrm{t}\right) \mathrm{T}\left(\mathrm{r}^{\prime}, \mathrm{a}^{\prime}, \mathrm{t}\right)=0 \Rightarrow \rho\left(\mathrm{r}, \mathrm{a}, \mathrm{r}^{\prime}, \mathrm{a}^{\prime}, \mathrm{t}\right)=0$.

Condition (ii) simply says that $\rho$ is a proportion. Condition (iii) states that the total number of pairs of $(r, a)$ individuals with $\left(r^{\prime}, a^{\prime}\right)$ individuals equals the total number of pairs of $\left(r^{\prime}, a^{\prime}\right)$ individuals with $(r, a)$ individuals (all this is per unit time, age, and time since infection). Condition (iv) says that there is no mixing in the age and activity levels at which there are no active individuals; i.e., on the set

$$
\boldsymbol{\varphi}=\left\{\left(\mathrm{r}, \mathrm{a}, \mathrm{r}^{\prime}, \mathrm{a}^{\prime}\right): \mathrm{C}(\mathrm{r}, \mathrm{a}, \mathrm{t}) \mathrm{T}(\mathrm{r}, \mathrm{a}, \mathrm{t}) \mathrm{C}\left(\mathrm{r}^{\prime}, \mathrm{a}^{\prime}, \mathrm{t}\right) \mathrm{T}\left(\mathrm{r}^{\prime}, \mathrm{a}^{\prime}, \mathrm{t}\right)=0\right\},
$$

where there is no mixing. Condition (iv) arises naturally in the study of the solutions of the above framework (see Busenberg and Castillo-Chavez 1990).

In some situations it is necessary to consider mixing functions $\rho$, which are Dirac delta functions or, more generally, distributions or generalized functions. Hence, we are forced to consider solutions to this axiomatic framework in the space of distributions or generalized functions (see Schwartz 1966, 
Gel'fand and Shilov 1964). To accomodate this possibility the following modification to the interpretation of axioms (i) and (iv) is necessary:

(i') $\rho \geq 0$ in the sense of distributions; i.e.,

$$
\int_{0}^{\infty} \int_{0} \rho\left(r, a, r^{\prime}, a^{\prime}, t\right) f\left(r^{\prime}, a^{\prime}, t\right) d r^{\prime} \mathrm{da}^{\prime} \geq 0 \text { for all } \mathrm{f} \geq 0 \text {, and }
$$

(iv') $\rho\left(\mathrm{r}, \mathrm{a}, \mathrm{r}^{\prime}, \mathrm{a}^{\prime}, \mathrm{t}\right)=0$ on a set $\mathrm{F}$, which means

$$
\int_{\mathrm{F}} \int \rho\left(\mathrm{r}, \mathrm{a}, \mathrm{r}^{\prime}, \mathrm{a}^{\prime}, \mathrm{t}\right) \mathrm{f}\left(\mathrm{r}, \mathrm{a}^{\prime}, \mathrm{t}\right) \mathrm{drda}^{\prime}=0 \text { for all } \mathrm{f} \text {. }
$$

Pair formations can involve selectivity by individuals according to age or activity level, they can be random pairings without regard to these variables, or they can be any combination or mixture of the two extremes. A detailed discussion of these possibilities and of the restrictions they place on the mixing function $\rho$ is found in Busenberg and Castillo-Chavez (1990).

A solution of critical importance to the mixing framework is that of total (i.e. in age and risk) proportionate mixing:

$$
\bar{\rho}\left(r, a, r^{\prime}, \mathbf{a}^{\prime}, t\right)=\frac{C\left(r^{\prime}, a^{\prime}, t\right) T\left(r^{\prime}, a^{\prime}, t\right)}{\iint_{0}^{\infty} C\left(r^{\prime}, a^{\prime}, t\right) T\left(r^{\prime}, a^{\prime}, t\right) d a^{\prime} d r^{\prime}} .
$$

This solution plays an important role in the determination of all possible solutions to the mixing framework (i)-(iv). Note that proportionate mixing vacuously satisfies condition (iv). This condition prevents us from accidentally dividing by zero, and hence prevents us from arbitrarily defining a mixing function for subpopulations that either are not sexually active or that have been depleted of individuals by disease dynamics. Further examples of specific mixing functions can be found in Castillo-Chavez and Blythe (1989) and Busenberg and Castillo-Chavez (1990). We further observe that convex linear combinations of mixing functions are mixing functions. Specifically, if $\alpha_{1}, \ldots \alpha_{N}$ are positive constants such that $\sum_{i=1}^{N} \alpha_{i}=1$ and $\rho_{1}, \ldots, \rho_{N}$ are mixing functions, then $\sum_{i=1}^{N} \alpha_{i} \rho_{i}$ is a mixing function. This last observation provides a recipe for the construction of a variety of mixing functions. Furthermore, it clearly shows that preferred mixing (a convex combination of two mixing functions), contrary to the suggestions of some researchers, does not contain all reasonable possibilities. Specifically, (omitting age) preferred mixing is given by

$$
\rho(\mathrm{s}, \mathrm{r})=(1-\alpha) \frac{\mathrm{C}(\mathrm{r}) \mathrm{T}(\mathrm{r})}{\int_{0}^{\infty} \mathrm{C}(\mathrm{u}) \mathrm{T}(\mathrm{u}) \mathrm{du}}+\alpha \delta(\mathrm{s}-\mathrm{r}),
$$

where $\delta$ denotes the Dirac delta (see Blythe and Castillo-Chavez 1989), i.e., it is the convex linear combination of the Dirac delta (a mixing function) and proportionate mixing. The two extreme points 
of this particular convex linear combination (when $\alpha=0$ or 1 ) do not obviously represent sociological or mathematical mixing extremes--this was pointed out to us by S. Gupta and R. Anderson.

A mixing function $\rho$ is called separable if it can be written in the form

$$
\rho\left(\mathrm{r}, \mathrm{a}, \mathbf{r}^{\prime}, \mathrm{a}^{\prime}, \mathrm{t}\right)=\rho_{1}(\mathrm{r}, \mathrm{a}, \mathrm{t}) \rho_{2}\left(\mathrm{r}^{\prime}, \mathrm{a}^{\prime}, \mathrm{t}\right) .
$$

The total proportionate mixing function $\bar{\rho}$ is separable, and our first result states that there are no other separable pairing functions.

Theorem 2.1 The only separable pairing function $\rho$ satisfying conditions (i)-(ii)-(iii) is the total proportionate mixing function $\bar{\rho}$ given by (5).

Proof: This result can be easily obtained by direct substitution of (3) into the mixing axioms. Since the proof of this result is similar to that of Theorem 3.1 (included later) we omit the details. .

All other solutions to the mixing framework are given by multiplicative perturbations of total proportionate mixing. The nature of the perturbations is specified in the following theorem:

Theorem 2.2 Let $\phi: \mathscr{B}_{+}^{4} \rightarrow \mathscr{B}_{B}$ be measurable and jointly symmetric: $\phi\left(\mathrm{r}, \mathrm{a}, \mathrm{r}^{\prime}, \mathrm{a}^{\prime}\right)=\phi\left(\mathrm{r}^{\prime}, \mathrm{a}^{\prime}, \mathrm{r}, \mathrm{a}\right)$, and suppose that

and

$$
\int_{0}^{\infty} \int_{\rho}^{\infty}\left(\mathbf{r}^{\prime}, \mathbf{a}^{\prime}\right) \phi\left(\mathbf{r}, \mathbf{a}, \mathbf{r}^{\prime}, \mathbf{a}^{\prime}\right) \mathrm{d} \mathbf{r}^{\prime} \mathrm{da} \mathbf{a}^{\prime} \leq 1,
$$

Let

$$
\int_{0}^{\infty} \int_{0} \bar{\rho}(\mathbf{r}, \mathbf{a})\left(\int_{0}^{\infty} \int_{0} \bar{\rho}\left(\mathbf{r}^{\prime}, \mathbf{a}^{\prime}\right) \phi\left(\mathbf{r}, \mathbf{a}, \mathbf{r}^{\prime}, \mathbf{a}^{\prime}\right) \mathrm{dr} \mathbf{r}^{\prime} \mathbf{d a} \mathbf{a}^{\prime}\right) \mathrm{drda}<1 .
$$

so that

$$
\rho_{1}(\mathrm{r}, \mathrm{a})=1-\int_{0}^{\infty} \int_{\rho} \bar{\rho}\left(\mathrm{r}^{\prime}, \mathrm{a}^{\prime}\right) \phi\left(\mathrm{r}, \mathrm{a}, \mathbf{r}^{\prime}, \mathrm{a}^{\prime}\right) \mathrm{d} \mathbf{r}^{\prime} \mathrm{da} \mathbf{a}^{\prime}
$$

$$
\rho\left(\mathbf{r}, \mathbf{a}, \mathbf{r}^{\prime}, \mathbf{a}^{\prime}\right)=\bar{\rho}\left(\mathbf{r}^{\prime}, \mathbf{a}^{\prime}\right) \llbracket \frac{\rho_{1}(\mathbf{r}, \mathbf{a}) \rho_{1}\left(\mathbf{r}^{\prime}, \mathbf{a}^{\prime}\right)}{\iint_{0}^{\infty} \bar{\rho}\left(\mathbf{r}^{\prime}, \mathbf{a}^{\prime}\right) \rho_{1}\left(\mathbf{r}^{\prime}, \mathbf{a}^{\prime}\right) d \mathbf{r}^{\prime} d \mathbf{a}^{\prime}}+\phi\left(\mathbf{r}, \mathbf{a}, \mathbf{r}^{\prime}, \mathbf{a}^{\prime}\right) \rrbracket
$$

is a mixing function. Conversely, for every mixing function $\rho$ there exists a $\phi$ that satisfies the hypotheses of the theorem such that $\rho$ is given by (5) with $\rho_{1}$ defined by (4).

Proof: That the expression given by Equation (5) is a mixing function is immediate. For the proof of the converse, see Busenberg and Castillo-Chavez (1990).

The function $\phi$ provides us with a measure of the deviation from proportionate mixing and therefore it is a measure of preference. We call this perturbation the structural covariance or preference function (note that this covariance is always positive). To illustrate the effects of $\phi$ on the shape of the 
mixing or pair formation function, we look at some examples for situations in which the mixing function is only a function of the age or risk (related to frequency and type of sexual activity) of the individuals but not of both. The version that is illustrated in our numerical simulations corresponds to the following version of Theorem 2.2:

Theorem 2.3 Let $\phi: \Re_{+}^{2} \rightarrow \Re^{+}$be a measurable and jointly symmetric function, and suppose that

$$
\begin{aligned}
& \int_{0}^{\infty} \bar{\rho}(\mathrm{r}) \phi\left(\mathrm{r}, \mathrm{r}^{\prime}\right) \mathrm{dr} \leq 1 \text { and } \\
& \int_{0}^{\infty} \bar{\rho}(\mathrm{r})\left\{\int_{0}^{\infty} \bar{\rho}(\mathrm{u}) \phi(\mathrm{u}) \mathrm{du}\right\} \mathrm{dr}<1 .
\end{aligned}
$$

Defining $\rho_{1}(\mathrm{r})$ by

$$
\rho_{1}(\mathrm{r})=1-\int_{0}^{\infty} \bar{\rho}(\mathrm{u}) \phi(\mathrm{r}, \mathrm{u}) \mathrm{du}
$$

we obtain the following representation formula for a two dimensional one-sex mixing function:

$$
\rho\left(\mathrm{r}, \mathrm{r}^{\prime}\right)=\bar{\rho}(\mathrm{r})\left[\frac{\rho_{1}(\mathrm{r}) \rho_{1}\left(\mathrm{r}^{\prime}\right)}{\int_{0}^{\infty} \bar{\rho}(\mathrm{r}) \rho_{1}(\mathrm{r}) \mathrm{dr}}+\phi\left(\mathrm{r}, \mathrm{r}^{\prime}\right)\right],
$$

where

$$
\bar{\rho}(\mathrm{r})=\frac{\mathrm{C}(\mathrm{r}) \mathrm{T}(\mathrm{r})}{\int_{0}^{\infty} \mathrm{C}(\mathrm{u}) \mathrm{T}(\mathrm{u}) \mathrm{du}}
$$

i.e., we have a multiplicative perturbation of proportionate mixing. Also for every mixing function $\rho$, there exists a structural covariance or preference function $\phi$ satisfying the hypotheses of the theorem such that $\rho$ is given by (7) with $\rho_{1}$ defined by (6).

We now proceed to illustrate the effects of preference on the shape of the mixing function. As a model for the distribution of activity levels in a population, the lognormal distribution has appeal due to its flexibility. Formally, if $\ln (R)$ has a normal distribution with mean $\mu$ and variance $\sigma^{2}$, then $R$ has a two-parameter lognormal distribution with parameters $\mu$, and $\sigma$. For convenience, define $b=$ $\mathrm{e}^{\mu}$. The probability density function for the lognormal may be written as 


$$
\mathrm{T}(r)=\frac{1}{\sigma r \sqrt{2 \pi}} \exp \left[-\frac{1}{2 \sigma^{2}}\left(\ln \left(\frac{r}{b}\right)\right)^{2}\right]=\operatorname{Prob}[\ln (\mathrm{R})=\mathrm{r}], r>0
$$

The mean and variance of $R$ are

$$
\mathrm{E}(R)=\mu_{R}=b \exp \left(\frac{\sigma^{2}}{2}\right)
$$

and

$$
\operatorname{Var}(R)=\sigma_{R}^{2}=b^{2} \mathrm{e}^{\sigma^{2}}\left(\mathrm{e}^{\sigma^{2}}-1\right)
$$

A more natural parameterization for our modeling purposes is to describe the distribution in terms of $\mu_{R}$ and $\sigma_{R}^{2}$. Given values of these two population parameters (either arbitrarily, or as suggested by data), we can easily determine that

and

$$
\sigma^{2}=\ln \left[\sigma_{R}^{2} \mu_{R}^{-1}+1\right]
$$

$$
b=\mu_{R} \exp \left(-0.5 \sigma^{2}\right) .
$$

We may further simplify our model prescription if we accept the empirical "power law" of Anderson and May (1988):

$$
\sigma_{R}^{2}=0.555 \mu_{R}^{3.231}
$$

whence

and

$$
\sigma^{2}=\ln \left[0.555 \mu_{R}^{2.231}+1\right]
$$

$$
b=\mu_{R} \exp \left(-0.5 \sigma^{2}\right) .
$$

With $\mathrm{C}(r)=r$, then $(8)$ becomes

$$
\bar{\rho}\left(\mathbf{r}^{\prime}\right)=\frac{\mathbf{r}^{\prime} \mathrm{T}\left(\mathrm{r}^{\prime}\right)}{\int_{0}^{\infty} \mathrm{uT}(\mathrm{u}) \mathrm{du}}
$$

where the denominator is the expected value of a lognormal random variable, i.e.,

$$
=\frac{1}{\sigma b \sqrt{2 \pi}} \exp -\left[\frac{1}{2 \sigma^{2}}\left(\ln \left(\frac{r^{\prime}}{b}\right)\right)^{2}+\left(\frac{\sigma^{2}}{2}\right)\right] .
$$

$T(r)$ is really a function of $t$, i.e., $T=T(r, t)$, and its behavior is governed by an appropriate partial differential equation (see Busenberg and Castillo-Chavez, 1989). Note however, that the "power law" of Anderson and May (1988) suggests that the mean and variance of $T(r, t)$ (regardless of how we model it) has to satisfy Equation (9). Further, since our purpose is to illustrate the effects of the structural covariance or preference function on the shape of $\rho\left(r, r^{\prime}, t\right)$, we "bypass" the dynamic model and concentrate on the effects of $\phi$ on $\rho$ when $\mu_{R}$ and $\sigma_{R}$ satisfy (9). Finally, we observe that a 
population that is experiencing a decrease in sexual activity will have to do it in a resticted fashion, i.e., by moving down along the line (in log-log scale) defined by Equation (9).

In our numerical illustrations we take a fairly general $\phi$, namely:

$$
\phi\left(r, r^{\prime}\right)=\exp \left[-\left(c_{1}\left(r^{2}+r^{2}\right)+c_{2} r r^{\prime}\right)\right]
$$

Recall that $\rho_{1}(r)=1-\int_{0}^{\infty} \bar{\rho}\left(r^{\prime}\right) \phi\left(r, r^{\prime}\right) d r^{\prime}$ must be $\leq 1$; and note that for our current choices for $\mathrm{T}$ and $\phi$, this condition is met for a wide range of $\mu_{R}, c_{1}$ and $c_{2}$, including values which may be reasonable for human populations. The denominator term of $\rho\left(\mathrm{r}, \mathrm{r}^{\prime}\right)$ is fairly cumbersome, but can be cleaned up a little:

$$
\begin{aligned}
& \int_{0}^{\infty} \bar{\rho}(\mathrm{r}) \rho_{1}(\mathrm{r}) \mathrm{d} \mathrm{r}=\int_{0}^{\infty} \frac{1}{\sigma b \sqrt{2 \pi}} \exp \left[\frac{-\left(\ln \left(r^{\prime} / b\right)\right)^{2}}{2 \sigma^{2}}-\left(\frac{\sigma^{2}}{2}\right)\right] \mathrm{d} r- \\
& \int_{0}^{\infty} \int_{0}^{\infty} \frac{\mathrm{c}_{1}}{2 \sigma^{2} b^{2} \pi} \exp -\left[\frac{\left(\ln \left(r^{\prime} / b\right)\right)^{2}}{\sigma^{2}}+\sigma^{2}+\mathrm{c}_{1}\left(r^{2}+r^{2}\right)+\mathrm{c}_{2} r r^{\prime}\right] \mathrm{d} r^{\prime} \mathrm{d} r
\end{aligned}
$$

The second term can be reduced to a one-dimensional integral by use of the change of variable defined by $u=\sqrt{2 c_{1}} r+\frac{c_{2} r}{\sqrt{2 c_{1}}}$. Then we have

$$
\int_{0}^{\infty} \frac{c_{1}}{2 \sigma^{2} b^{2} \sqrt{c_{1} \pi}} \exp \left[-\left(\frac{\left(\ln \left(r^{\prime} / b\right)\right)}{\sigma}\right)^{2}-\sigma^{2}-{r^{\prime}}^{2}\left(c_{1}-\frac{c_{2}^{2}}{4 c_{1}}\right)\right]\left(1-\Phi\left(\frac{c_{2} r^{\prime}}{\sqrt{2 c_{1}}}\right)\right) d r^{\prime}, \text { where }
$$

$\Phi(\cdot)$ is the standardized Gaussian cumulative distribution function.

In our set of simulations, we use all six combinations of two choices for $T(r)$ (determined by the Anderson and May's (1988) power law, with values of 2 and 8 for $\mu_{R}$ ) and three choices for $\phi: \phi_{1}$ with a well-defined narrow ridge along the line $r=r^{\prime}$ (determined by the pair $\left.\left(c_{1}, c_{2}\right)=(0.3,-0.6)\right), \phi_{2}$ with a somewhat broader profile $\left(\left(c_{1}, c_{2}\right)=(0.05,-0.08)\right)$, and $\phi_{3}=0$, representing proportional mixing. We have plots also of the corresponding structural covariance functions $\phi_{1}$ and $\phi_{2}$.

The plots illustrate the interaction between the structural covariance function and the degree to which the population exhibits proportionate mixing. For a given mean activity level ( 2 and 8 in these simulations) the preference function exhibiting the sharpest degree of preference $\left(\phi_{1}\right.$, plot 1$)$ has the mixing function which is (visually, at least) furthest removed from proportionate mixing (plots 2, 3). As the preference function gets less sharply peaked $\left(\phi_{2}\right.$, plot 4$)$, the mixing function (plots 5,6$)$ is more similar to proportionate mixing (plot 7,8 ). Also, for a given $\phi$, as the population mean activity level 
increases, the mixing function looks more and more like a simple additive combination of $\phi$ with proportionate mixing (plots $2 \rightarrow 3,5 \rightarrow 6$ ).

\section{Two-sex mixing framework}

In this section we provide an outline of our two-sex framework. Since an extensive account will be provided later (see Castillo-Chavez and Busenberg, 1990), we look exclusively at our mixing framework in the context of a two-sex age-structured population. We further concentrate on a framework suitable for a two-sex demographic model. The modifications needed to transform this demographic model into an epidemiological model for sexually-transmitted diseases are straightforward and can be found in Castillo-Chavez and Busenberg (1990).

We let $M(a, t)$ denote the density of males of age a who are not in pairs at time $t$, and let $F\left(a^{\prime}, t\right)$ denote the density of females of age $a^{\prime}$ who are not in pairs at time $t$. Pairing is defined through the mixing functions:

$$
\begin{aligned}
p\left(a, a^{\prime}, t\right)= & \text { proportion of partnerships of males of age } a \text { with } \\
& \text { females of age } a^{\prime} \text { at time } t, \\
q\left(a, a^{\prime}, t\right)= & \text { proportion of partnerships of females of age } a^{\prime} \text { with } \\
& \text { males of age a at time } t,
\end{aligned}
$$

and we let

$$
\begin{aligned}
C(a, t)= & \text { expected or average number of partners of a male of } \\
& \text { age a at time } t \text { per unit time, } \\
D\left(a^{\prime}, t\right)= & \text { expected or average number of partners of a female of } \\
& \text { age } a^{\prime} \text { at time } t \text { per unit time, }
\end{aligned}
$$

The following natural conditions characterize these mixing functions:

(a) $p, q \geq 0$,

(b) $\int_{0}^{\infty} \mathrm{p}\left(\mathrm{a}, \mathrm{a}^{\prime}, \mathrm{t}\right) \mathrm{da^{ \prime }}=1=\int_{0}^{\infty} \mathrm{q}\left(\mathrm{a}^{\prime}, \mathrm{a}, \mathrm{t}\right) \mathrm{da}=1$,

(c) $\mathrm{p}\left(\mathrm{a}, \mathrm{a}^{\prime}, \mathrm{t}\right) \mathrm{C}(\mathrm{a}, \mathrm{t}) \mathrm{M}(\mathrm{a}, \mathrm{t})=\mathrm{q}\left(\mathrm{a}^{\prime}, \mathrm{a}, \mathrm{t}\right) \mathrm{D}\left(\mathrm{a}^{\prime}, \mathrm{t}\right) \mathrm{F}\left(\mathrm{a}^{\prime}, \mathrm{t}\right)$,

(d) $C(a, t) M(a, t) D\left(a^{\prime}, t\right) F\left(a^{\prime}, t\right)=0 \Rightarrow p\left(a, a^{\prime}, t\right)=q\left(a^{\prime}, a, t\right)=0$,

Condition (ii) is due to the fact that $\mathbf{p}$ and $\mathbf{q}$ are proportions. Condition (iii) simply states 
that the total number of pairs of males of age a with females of age $a^{\prime}$ equals the total number of pairs of females of age $a^{\prime}$ with males of age a (all per unit time and age). Condition (iv) says that there is no mixing in the age and activity levels where there are no active individuals; i.e., on the set $\varphi(t)=\left\{\left(a, a^{\prime}, t\right): C(r, a, t) M(a, t) D\left(a^{\prime}, t\right) F\left(a^{\prime}, t\right)=0\right\}$.

The pair $(p, q)$ is called a two-sex mixing function iff it satisfies axioms (a-d). Further, a two-sex mixing function is called separable iff

$$
p\left(a, a^{\prime}, t\right)=p_{1}(a, t) p_{2}\left(a^{\prime}, t\right) \quad \text { and } \quad q\left(a, a^{\prime}, t\right)=q_{1}(a, t) q_{2}\left(a^{\prime}, t\right)
$$

If we let

$$
h_{p}(a, t)=C(a, t) M(a, t)
$$

and

$$
\mathrm{h}_{\mathrm{q}}(\mathrm{a}, \mathrm{t})=\mathrm{D}(\mathrm{a}, \mathrm{t}) \mathrm{F}(\mathrm{a}, \mathrm{t})
$$

then, omitting $t$ to simplify the notation, we establish the following result:

Theorem 3.1 The only two-sex separable mixing function satisfying conditions (a-d) is given by $(\overline{\mathrm{p}}, \overline{\mathrm{q}})$, where

$$
\begin{aligned}
\bar{p}\left(a^{\prime}\right) & =\frac{h_{q}\left(a^{\prime}\right)}{\int_{0}^{\infty} h_{p}(n) d n}, \\
\bar{q}(a) & =\frac{h_{p}(a)}{\int_{0}^{\infty} h_{q}(n) d n}
\end{aligned}
$$

Proof: It is clear that the expressions given by Equations (13-14) satisfy the axioms (a-d), and hence, $(p, q)$ is a two-sex mixing function. Let's now assume that $(p, q)$ is separable, then using Axiom (b), we see that

$$
\left.\mathrm{p}_{1}(\mathrm{a})=\frac{1}{\int_{0}^{\infty} \mathrm{p}_{2}(\mathrm{n}) \mathrm{dn}}=\ell, \quad \text { (a constant }\right)
$$

and

therefore,

$$
q\left(a^{\prime}\right)=\frac{1}{\int_{0}^{\infty} q_{2}(n) d n}=k, \quad(a \text { constant })
$$

$$
\mathrm{p}\left(\mathrm{a}, \mathrm{a}^{\prime}\right)=\ell \mathrm{p}_{2}\left(\mathrm{a}^{\prime}\right) \quad \text { and } \quad \mathrm{q}\left(\mathrm{a}, \mathrm{a}^{\prime}\right)=\mathrm{k} \mathrm{q} \mathrm{q}_{2}(\mathrm{a})
$$


If we substitute the above expressions into Axiom (c) and integrate over all ages a, then we arrive at

$$
\ell \mathrm{p}_{2}\left(\mathrm{a}^{\prime}\right) \int_{0}^{\infty} \mathrm{h}_{\mathrm{p}}(\mathrm{n}) \mathrm{dn}=\mathrm{h}_{\mathrm{q}}(\mathrm{a})
$$

from which (13) follows. Equation (14) is obtained similarly.

Castillo-Chavez and Busenberg (1990), have established that all two-sex mixing functions are multiplicative perturbations (with appropriate structural covariance functions) of the only separable two-sex mixing function given by (13-14). Although, the general solution may prove to be quite useful in theoretical considerations, it is still of practical importance to provide modelers and theoreticians with flexible families of two-sex mixing functions. The following two-sex biased mixing family for $\mathrm{N}$ interacting subpopulations may fulfill this need. To introduce it, we let $u_{i}\left(v_{i}\right)$ denote the proportion of partnerships by males (females) of group i reserved for mixing with females (males) in group i; necessarily $0 \leq u_{i}, v_{i} \leq 1$. If $F_{i}(t)\left(M_{i}(t)\right)$ denote the number of males (females) in group $i$ at time $t$, and $C_{i}\left(D_{i}\right)$ denote the average number of female (male) sexual partners of males (females) in group $i$, and $p_{i j}(t)\left(q_{i j}(t)\right)$ denote the proportion of partnerships of males (females) in group $i$ with females (males) in group $\mathrm{j}$. Then

where

$$
\begin{array}{ll}
\mathrm{p}_{\mathrm{ij}}(\mathrm{t})=\mathrm{u}_{\mathrm{i}} \delta_{\mathrm{ij}}+\left(1-\mathrm{u}_{\mathrm{i}}\right) & \frac{\left(1-\mathrm{v}_{\mathrm{j}}\right) \mathrm{D}_{\mathrm{j}} \mathrm{F}_{\mathrm{j}}}{\sum_{\mathrm{k}=1}^{N}\left(1-\mathrm{v}_{\mathrm{k}}\right) \mathrm{D}_{\mathrm{k}} \mathrm{F}_{\mathrm{k}}}, \\
\mathrm{q}_{\mathrm{ji}}(\mathrm{t})=\mathrm{v}_{\mathrm{j}} \delta_{\mathrm{ji}}+\left(1-\mathrm{v}_{\mathrm{j}}\right) & \frac{\left(1-\mathrm{u}_{\mathrm{i}}\right) \mathrm{C}_{\mathbf{i}} \mathrm{M}_{\mathbf{i}}}{\sum_{\mathrm{k}=1}^{N}\left(1-\mathrm{u}_{\mathrm{k}}\right) \mathrm{C}_{\mathrm{k}} \mathrm{M}_{\mathrm{k}}},
\end{array}
$$

$$
\delta_{i j}= \begin{cases}1 & \text { if } i=j \\ 0 & \text { if } i \neq j\end{cases}
$$

The above family of two-sex biased mixing functions is easily incorporated into classical epidemiological models as well as into models that follow pairs. This is the topic of the next section where we introduce the simplest demographic model that follows pairs and that makes use of the framework of this section.

4. Demographic pair formation models

Demographic models that consider pairs and follow the dynamics of pairs have been studied 
by Kendall (1949), Fredrickson (1971), Dietz and Hadeler (1988), Dietz (1988), Hadeler (1989a,b), and Waldstätter (1989). Their approach is based on the use of a nonlinear function $\psi$ to model the process (rate) of pair formation. This mixing/pair formation function is assumed to satisfy the Fredrickson/McFarland $(1971,1972)$ properties:

$$
\psi(0, \mathrm{~F})=\psi(\mathrm{M}, 0)=0
$$

In the absence of either males or females there will be not heterosexual pair formation. $\psi(\alpha \mathrm{M}, \alpha \mathrm{F})=\alpha \psi(\mathrm{M}, \mathrm{F})$ for all $\alpha, \mathrm{M}, \mathrm{F} \geq 0$.

If the sex ratio remains constant, then the increase in the rate of pair formation is assumed to be proportional to total population size. $\psi(\mathrm{M}+\mathrm{u}, \mathrm{F}+\mathrm{v}) \geq \psi(\mathbf{M}, \mathrm{F})$ for all $\mathrm{u}, \mathrm{v}, \mathrm{F}, \mathrm{M} \geq \mathbf{0}$.

Increases in the number of males and/or females does not decrease the rate of pair formation.

Condition (f) implies that all mixing functions are of the form

$$
\psi(M, F)=M g\left(\frac{F}{M}\right)=F h\left(\frac{M}{F}\right),
$$

where $h$ and $g$ are functions of one-variable.

Mixing functions satisfying the above axioms, and that have been used in demographic studies, include:

$$
\begin{aligned}
& \psi(\mathrm{M}, \mathrm{F})=\mathrm{k} \min (\mathrm{M}, \mathrm{F}), \quad \mathrm{k} \text { is a constant } \\
& \psi(\mathrm{M}, \mathrm{F})=\mathrm{k} \sqrt{\mathrm{MF}}
\end{aligned}
$$

and

$$
\psi(\mathrm{M}, \mathrm{F})=2 \mathrm{k} \frac{\mathrm{MF}}{\mathrm{M}+\mathrm{F}} .
$$

Let $\sigma$ denote the rate of pair dissolution, $\mu$ denote the natural mortality rate, $\Lambda$ denote the "recruitment" rate, and $\mathrm{W}$ denote the number of (heterosexual) pairs. Then a simple demographic model is given by the following set of equations:

$$
\begin{aligned}
& \frac{\mathrm{dM}}{\mathrm{dt}}=\Lambda-\mu \mathrm{M}+(\sigma+\mu) \mathrm{W}-\psi(\mathrm{M}, \mathrm{F}) \\
& \frac{\mathrm{dF}}{\mathrm{dt}}=\Lambda-\mu \mathrm{F}+(\sigma+\mu) \mathrm{W}-\psi(\mathrm{M}, \mathrm{F})
\end{aligned}
$$




$$
\frac{\mathrm{dW}}{\mathrm{dt}}=(\sigma+2 \mu) \mathrm{W}+\psi(\mathrm{M}, \mathrm{F})
$$

If $\Lambda, \mu$, and $\sigma$, are constant, then there is always a globally stationary solution $(\mathrm{M}, \mathrm{F}, \mathrm{W})$, where $\mathrm{W}$ is determined by the equation

$$
\psi\left(\frac{\Lambda}{\mu}-\mathrm{W}, \frac{\Lambda}{\mu}+\mathrm{W}\right)=(\sigma+2 \mu) \mathrm{W} .
$$

(for references to this and related results see Waldstätter, 1989).

If we now let $f\left(a^{\prime}, t\right)$ and $m(a, t)$ denote the age-specific densities for single males and single females respectively, and assume that $D$ (as defined in Section 3) and $\mu_{\mathrm{m}}$ and $\mu_{\mathrm{f}}$ are functions of age (the mortality rates for males and females), and assume that $W\left(a, a^{\prime}, t\right)$ denotes the age-specific density of heterosexual pairs (where a denotes the age of the male and $a^{\prime}$ the age of the female), then using the two-sex mixing functions $p$ and $q$ of Section 3 , we arrive at the following demographic model for heterosexual populations:

$$
\begin{gathered}
\frac{\partial \mathrm{m}}{\partial \mathrm{t}}+\frac{\partial \mathrm{m}}{\partial \mathrm{a}}=-\mathrm{C}(\mathrm{a}) \mathrm{m}(\mathrm{a}, \mathrm{t}) \int_{0}^{\infty} \mathrm{p}\left(\mathrm{a}, \mathrm{a}^{\prime}, \mathrm{t}\right) \mathrm{d \textrm {a } ^ { \prime }} \\
\quad-\mu_{\mathrm{m}}(\mathrm{a}) \mathrm{m}(\mathrm{a}, \mathrm{t})+\int_{0}^{\infty}\left[\mu_{\mathrm{f}}\left(\mathrm{a}^{\prime}\right)+\sigma\right] \mathrm{W}\left(\mathrm{a}, \mathrm{a}^{\prime}, \mathrm{t}\right) \mathrm{da^{ \prime }} \\
\frac{\partial \mathrm{f}}{\partial \mathrm{t}}+\frac{\partial \mathrm{f}}{\partial \mathrm{a}^{\prime}}=-\mathrm{D}\left(\mathrm{a}^{\prime}\right) \mathrm{f}\left(\mathrm{a}^{\prime}, \mathrm{t}\right) \int_{0}^{\infty} \mathrm{q}\left(\mathrm{a}^{\prime}, \mathrm{a}, \mathrm{t}\right) \mathrm{da}
\end{gathered}
$$

To complete this model we need to specify the initial and boundary conditions. To this effect we let $\lambda_{m}$ and $\lambda_{f}$ denote the female-age-specific fertility rates, and let $m_{0}, f_{o}$, and $w_{0}$ denote the intial age densities. Hence, the initial and boundary conditions are given by

$$
\begin{aligned}
\mathrm{m}(0, \mathrm{t}) & =\int_{0}^{\infty} \lambda_{\mathrm{m}}\left(\mathrm{a}^{\prime}\right) \mathrm{W}\left(\mathrm{a}, \mathrm{a}^{\prime}, \mathrm{t}\right) \mathrm{da}^{\prime}, \\
\mathrm{f}(0, \mathrm{t}) & =\int_{0}^{\infty} \lambda_{\mathrm{f}}\left(\mathrm{a}^{\prime}\right) \mathrm{W}\left(\mathrm{a}, \mathrm{a}^{\prime}, \mathrm{t}\right) \mathrm{da}^{\prime}
\end{aligned}
$$




$$
\begin{aligned}
W(0,0, t) & =0 \\
f(a, 0) & =f_{o}(a), m(a, 0)=m_{0}(a), \quad W\left(a, a^{\prime}, 0\right)=W_{0}\left(a, a^{\prime}\right) .
\end{aligned}
$$

Let

$$
N_{f}\left(a^{\prime}, t\right)=\int_{0}^{\infty} W\left(a, a^{\prime}, t\right) d a,
$$

and observe that $N_{f}$ and $f+N_{f}$ satisfy the following set of equations:

$$
\left(\frac{\partial}{\partial t}+\frac{\partial}{\partial a^{\prime}}\right)\left(f+N_{f}\right)=-\mu_{f}\left(a^{\prime}\right)\left[f+N_{f}\right]
$$

and

$$
\begin{aligned}
&\left(\frac{\partial}{\partial t}+\frac{\partial}{\partial a^{\prime}}\right) N_{f}=D\left(a^{\prime}\right) f\left(a^{\prime}, t\right) \\
&-\left[\mu_{f}\left(a^{\prime}\right)+\sigma\right] N_{f} \\
&-\int_{0}^{\infty} \mu_{m}(a) Q\left(a, a^{\prime}, t\right) d a .
\end{aligned}
$$

If we assume that $\mu_{\mathrm{m}}, \mu_{\mathrm{f}}$ and $\mathrm{D}$ are constants and look for solutions of the form

$$
N_{f}\left(a^{\prime}, t\right)=e^{r t} \tilde{N}\left(a^{\prime}\right)
$$

and

$$
f\left(a^{\prime}, t\right)=e^{r t} \tilde{f}(a)
$$

then in the usual fashion, we arrive at a characteristic equation for $r$ of the the form

$$
\mathrm{H}(\mathbf{r})=1
$$

where

$$
\mathrm{H}(\mathrm{r})=\int_{0}^{\infty} \lambda_{\mathrm{f}}(\mathrm{a}) \frac{\mathrm{D}}{\mu_{\mathrm{m}}+\mathrm{D}+\sigma} \mathrm{e}^{-\left(\mathrm{r}+\mu_{\mathrm{f}}\right) \mathrm{a}}\left[1-\mathrm{e}^{-\left(\mu_{\mathrm{m}}+\sigma+\mathrm{D}\right) \mathrm{a}}\right] \mathrm{da} .
$$

Since $H(r)$ is decreasing, then (25) has a unique real root $\mathbf{r}_{*}$. Clearly

$$
\mathrm{H}(0)>0 \Leftrightarrow \mathrm{r}_{*}>0 \text {, }
$$

and if $\mathrm{r}=\alpha+\mathrm{i} \beta$ is a complex root then one easily sees that $\alpha<\mathrm{r}_{*}$. We observe further that the above analysis is independent of $\rho$, however, note that in order to recover $W$ or to study the stability of 
product solutions we need to have specific knowledge of the mixing function $\rho$.

Although further analysis is possible, we will not present it here, as one of the main purposes of this article is to show an alternative approach to that of Fredrickson/McFarland/Dietz/Hadeler for the formulation of demographic models that follow the dynamics of pairs. Epidemiological models that fit into our framework are easily formulated and the appropriate details will be discussed elsewhere.

\section{Conclusions}

In this article we have presented a general solution to the one sex mixing/pair formation problem. Our representation theorem states that any mixing function can be represented as a multiplicative perturbation of proportionate mixing. This perturbation, through its structural covariance or preference function, provides us with a measure of divergence from proportionate mixing. Simulations based on the "power law" of Anderson and May (1988) were provided to illustrate the role of preference in the shape of the mixing function. Our discussion of the simulation results, combined with our previous studies (see Blythe and Castillo-Chavez 1989, Castillo-Chavez and Blythe 1989, Busenberg and Castillo-Chavez 1989), show that to understand the role of preference in disease dynamics we need to develop methods of estimating the effects of the structural covariance function on the shape of the basic mixing function (i.e. proportionate mixing). Knowledge of "realistic" mixing structures is needed in the evaluation of possible intervention programs aimed at disease prevention.

We have also introduced a two-sex mixing framework and constructed a variety of solutions that may prove useful in applications. Finally, we have introduced a demographic model that follows pairs based on our mixing/pair-formation framework, and have shown that this model has nontrivial solutions. Further analysis of this model is being carried out and will be published elsewhere.

\section{ACKNOWLEDGMENTS}

This research has been partially supported by the Center for Applied Mathematics at Cornell University and NSF grant DMS-8703631 to Stavros Busenberg, and by NSF grant DMS-8906580, NIAID Grant R01 A129178-01, and Hatch project grant NYC 151-409, USDA to Carlos CastilloChavez. We thank S. P. Blythe for his valuable comments. 


\section{REFERENCES}

Anderson, R.M. (1988). "The role of mathematical models in the study of HIV transmission and the epidemiology of AIDS.” Journal of AIDS. 1:241-256.

Anderson, R.M. (1989). Editorial Review: Mathematical and Statistical Studies of the epidemiology of HIV. AIDS. 3:333-346

Anderson, R.M. and R. M. May (1988). Epidemiological parameters of HIV transmission. Nature. 333: 514-519

Anderson, R. M., S. P. Blythe, S. Gupta, and E. Konings. (1989). The transmission dynamics of the Human Immunodeficiency Virus Type 1 in the male homosexual community in the United Kindom: the influence of changes in sexual behavior. (Manuscript.)

Blythe, S.P. and C. Castillo-Chavez. (1989). Like-with-like preference and sexual mixing models. Math. Biosci. 96, 221-238.

Busenberg, S. and C. Castillo-Chavez. (1989). Interaction, pair formation and force of infection terms in sexually transmitted diseases. In (C. Castillo-Chavez, ed.) Mathematical and Statistical Approaches to AIDS Epidemiology. Lecture Notes in Biomathematics 83, Springer-Verlag, Berlin, Heidelberg, New York, London, Paris, Tokyo, Hong Kong: 289-300

Busenberg, S. and C. Castillo-Chavez. (1990). On the role of preference in the solution of the mixing problem, and its application to risk- and age- structured epidemic models. (Submitted to IMA J. of Math. Applic. to Med. and Biol.)

Castillo-Chavez, C. (1989a). Review of recent models of HIV/AIDS transmission. In (S. A. Levin, T. G. Hallam, and L. J. Gross, eds.) Applied Mathematical Ecology, Biomathematics 18, SpringerVerlag, Berlin, Heidelberg, New York, London, Paris, Tokyo, Hong Kong: 253-262

Castillo-Chavez, C. (ed.). (1989b). Mathematical and Statistical Approaches to AIDS Epidemiology. Lecture Notes in Biomathematics 83, Springer-Verlag, Berlin, Heidelberg, New York, London, Paris, Tokyo, Hong Kong.

Castillo-Chavez, C. and S.P. Blythe. (1989). Mixing framework for social/sexual behavior. In (C. Castillo-Chavez, ed.) Mathematical and Statistical Approaches to AIDS Epidemiology. Lecture Notes in Biomathematics 83, Springer-Verlag, Berlin, Heidelberg, New York, London, Paris, Tokyo, Hong Kong: 275-288

Castillo-Chavez, C. and S. Busenberg. (1990). Pair formation in age- and risk-structured populations. (Manuscript in preparation.)

Dietz, K. (1988). On the transmission dynamics of HIV. Math. Biosci. 90, 397-414. 
Dietz, K. and K.P. Hadeler. (1988). Epidemiological models for sexually transmitted diseases. J. Math. Biol. 26, 1-25.

Fredrickson, A.G. (1971). A mathematical theory of age structure in sexual populations: Random mating and monogamous marriage models. Math. Biosci. 20, 117-143.

Gel'fand, I. M. and G. E. Shilov. (1964). Generalized Functions. Academic Press, New York, London. Volume 1.

Hadeler, K.P. (1989a). Pair formation in age-structured populations. Acta Applicandae Mathematicae 14, 91-102.

Hadeler, K.P. (1989b). Modeling AIDS in structured populations. (Manuscript.)

Kendall, D.G. (1949). Stochastic processes and population growth. Roy. Statist. Soc., Ser. B 2, 230264.

McFarland, D. D. (1972). Comparison of alternative marriage models. In (Greville, T. N. E., ed.), Population Dynamics. Academic Press, New York London: 107-135.

Sattenspiel, L. (1987a). Population structure and the spread of disease. Human Biology 59, 411-438.

Sattenspiel, L. (1987b). Epidemics in nonrandomly mixing populations: a simulation. American Journal of Physical Anthropology, 73, 251-265.

Sattenspiel, L. and C.P. Simon. (1988). The spread and persistence of infectious diseases in structured populations. Math. Biosci. 90, 341-366.

Schwager, S. J., C. Castillo-Chavez, and H. Hethcote. (1989). Statistical and mathematical approaches in HIV/AIDS modeling: a review. In (C. Castillo-Chavez, ed.) Mathematical and Statistical Approaches to AIDS Epidemiology. Lecture Notes in Biomathematics 83, SpringerVerlag, Berlin, Heidelberg, New York, London, Paris, Tokyo, Hong Kong: 2-37

Schwartz, L. (1966). Théorie des distributions. Hermann, Paris.

Waldstätter, R. (1989). Pair formation in sexually transmitted diseases. In (C. Castillo-Chavez, ed.) Mathematical and Statistical Approaches to AIDS Epidemiology. Lecture Notes in Biomathematics 83, Springer-Verlag, Berlin, Heidelberg, New York, London, Paris, Tokyo, Hong Kong: 260-274 
Plot 1: $\phi$, with $\mathrm{c}_{1}=0.3, \mathrm{c}_{2}=\mathbf{- 0 . 6}$.

Plot 2: $\rho$, with $\mu_{\mathrm{R}}=2, c_{1}=0.3, c_{2}=-0.6$.

Plot 3: $\rho$, with $\mu_{\mathrm{R}}=8, \mathrm{c}_{1}=0.3, \mathrm{c}_{2}=-0.6$.

Plot 4: $\phi$, with $c_{1}=0.05, c_{2}=-0.08$.

Plot 5: $\rho$, with $\mu_{\mathrm{R}}=2, \mathrm{c}_{1}=0.05, \mathrm{c}_{2}=-0.08$.

Plot 6: $\rho$, with $\mu_{\mathrm{R}}=8, \mathrm{c}_{1}=0.05, \mathrm{c}_{2}=-0.08$.

Plot 7: $\rho$, with $\mu_{\mathrm{R}}=2, \phi=0$.

Plot 8: $\rho$, with $\mu_{\mathrm{R}}=4, \phi=0$. 


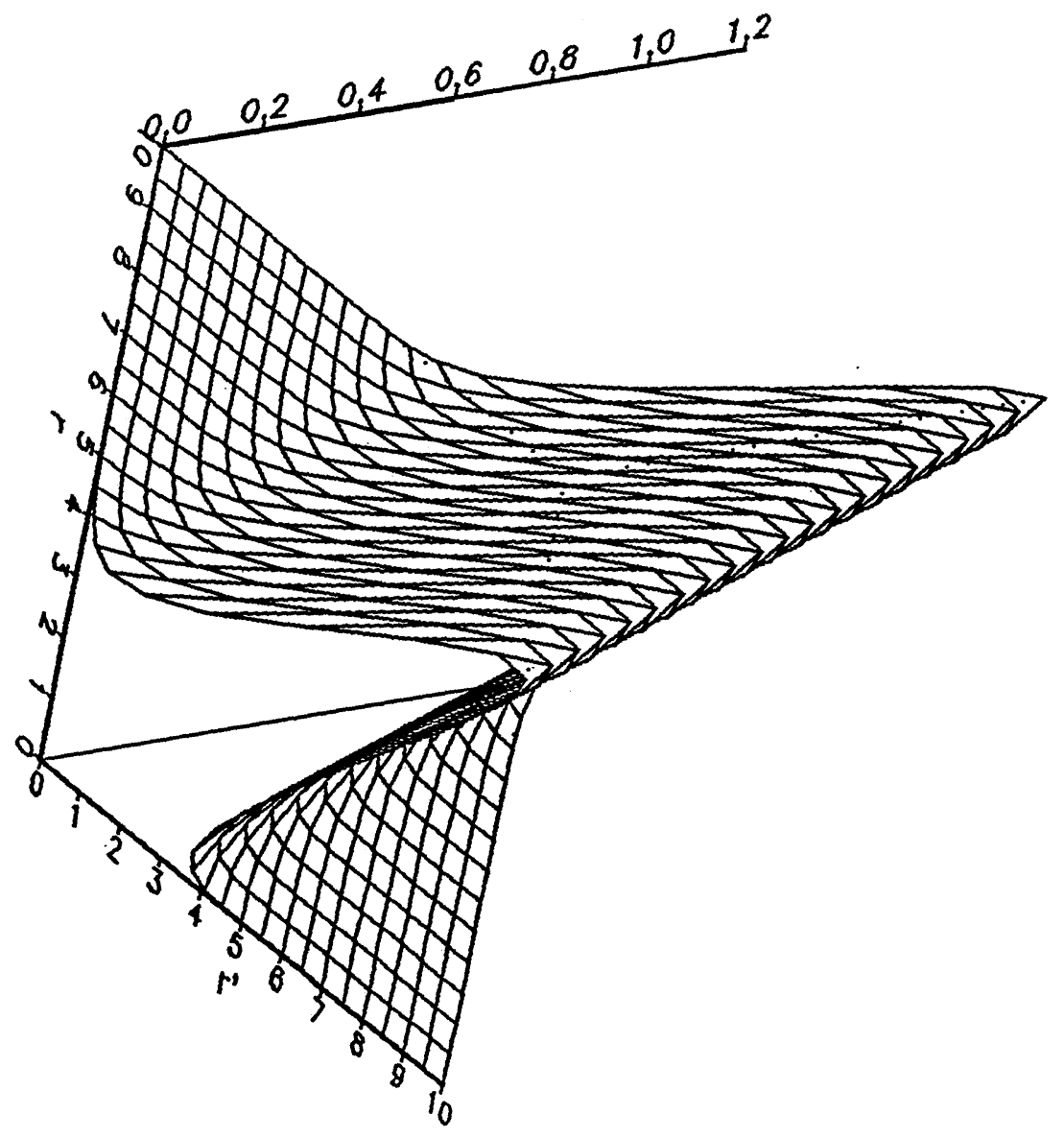




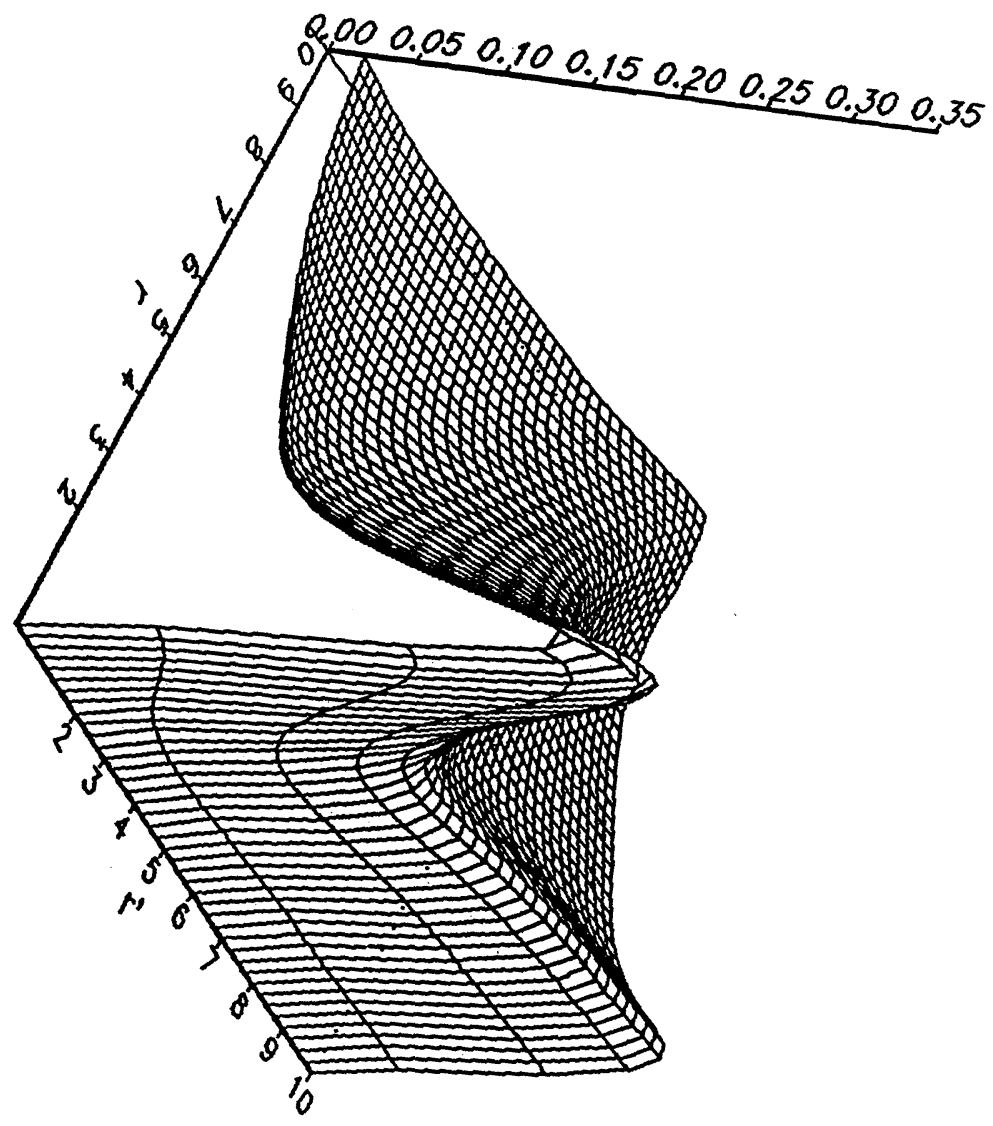




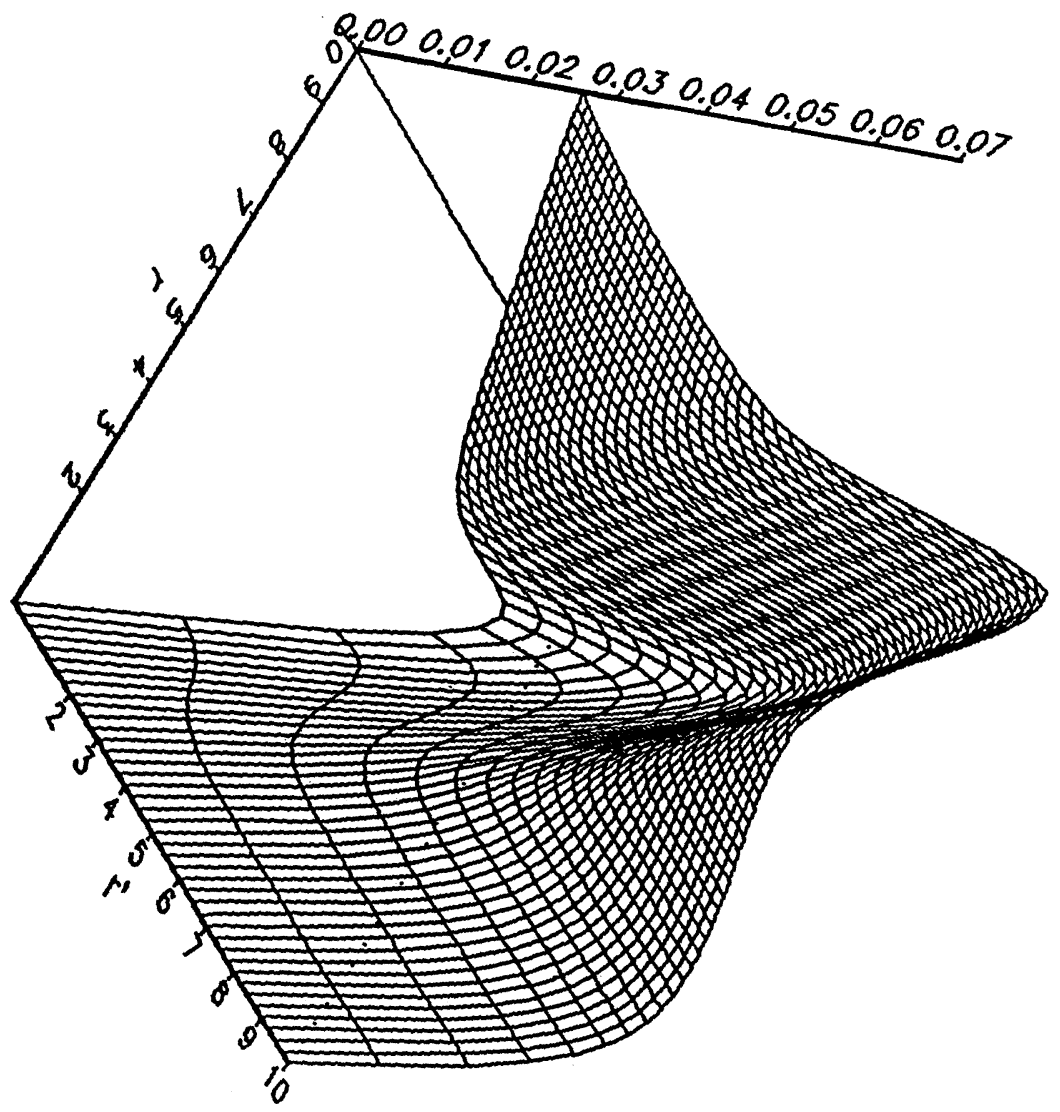




$$
\diamond
$$




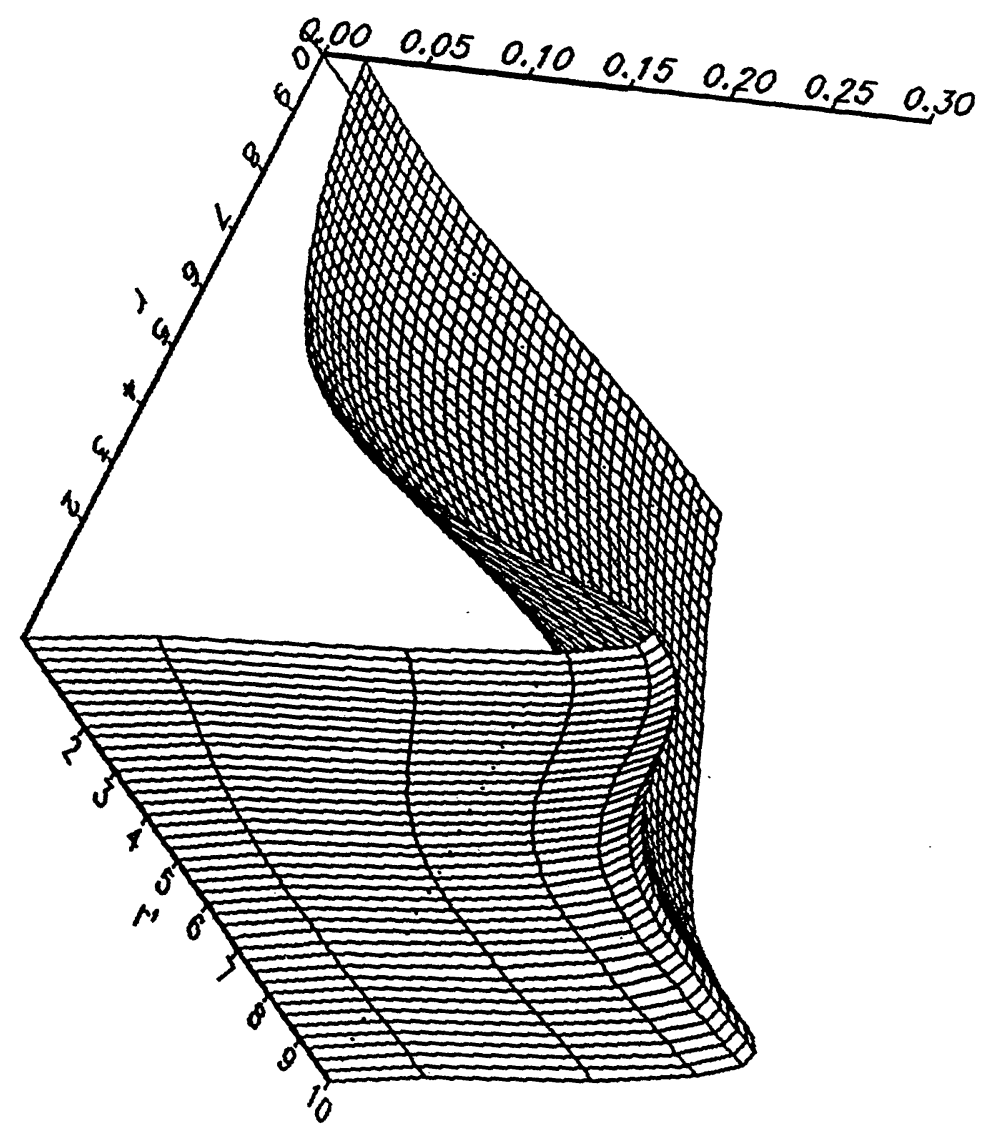




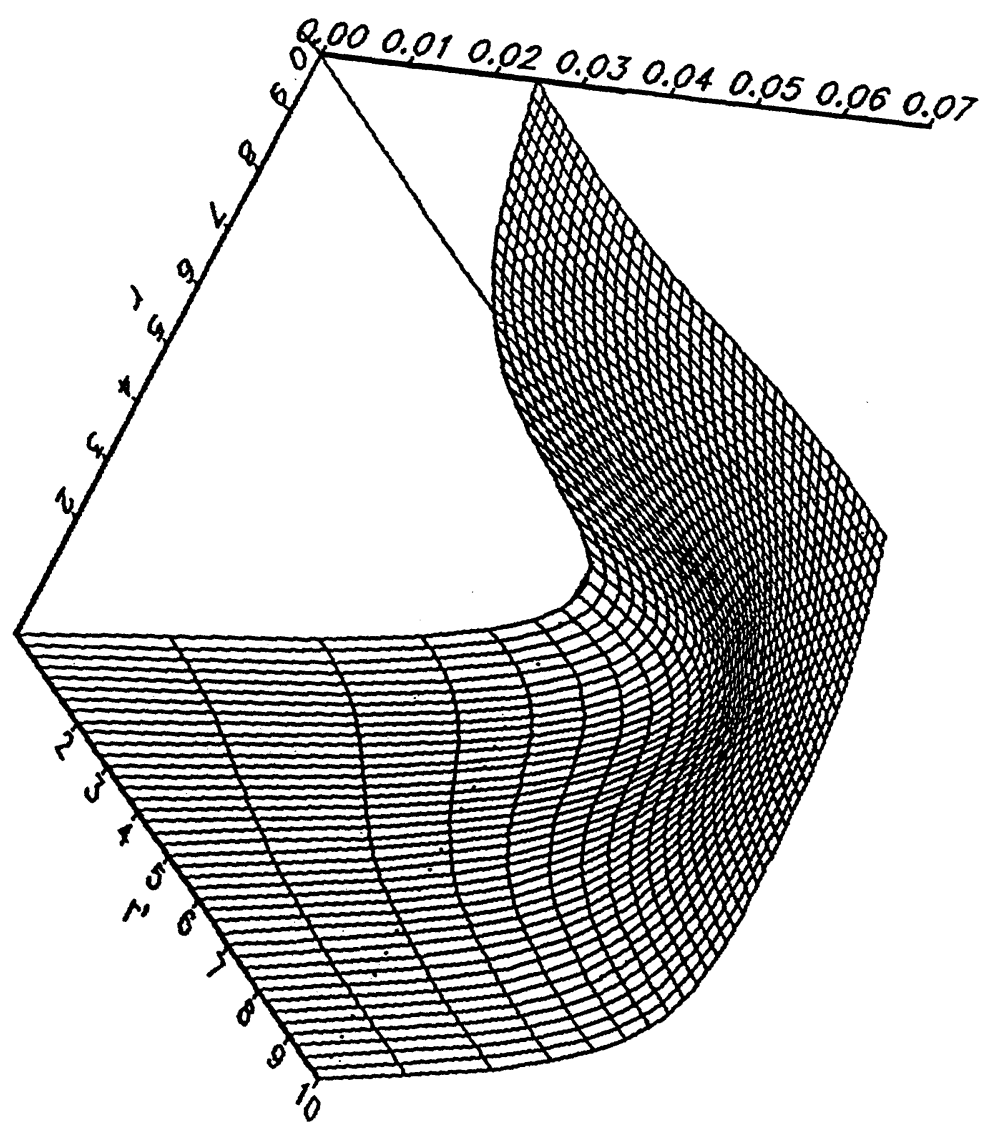




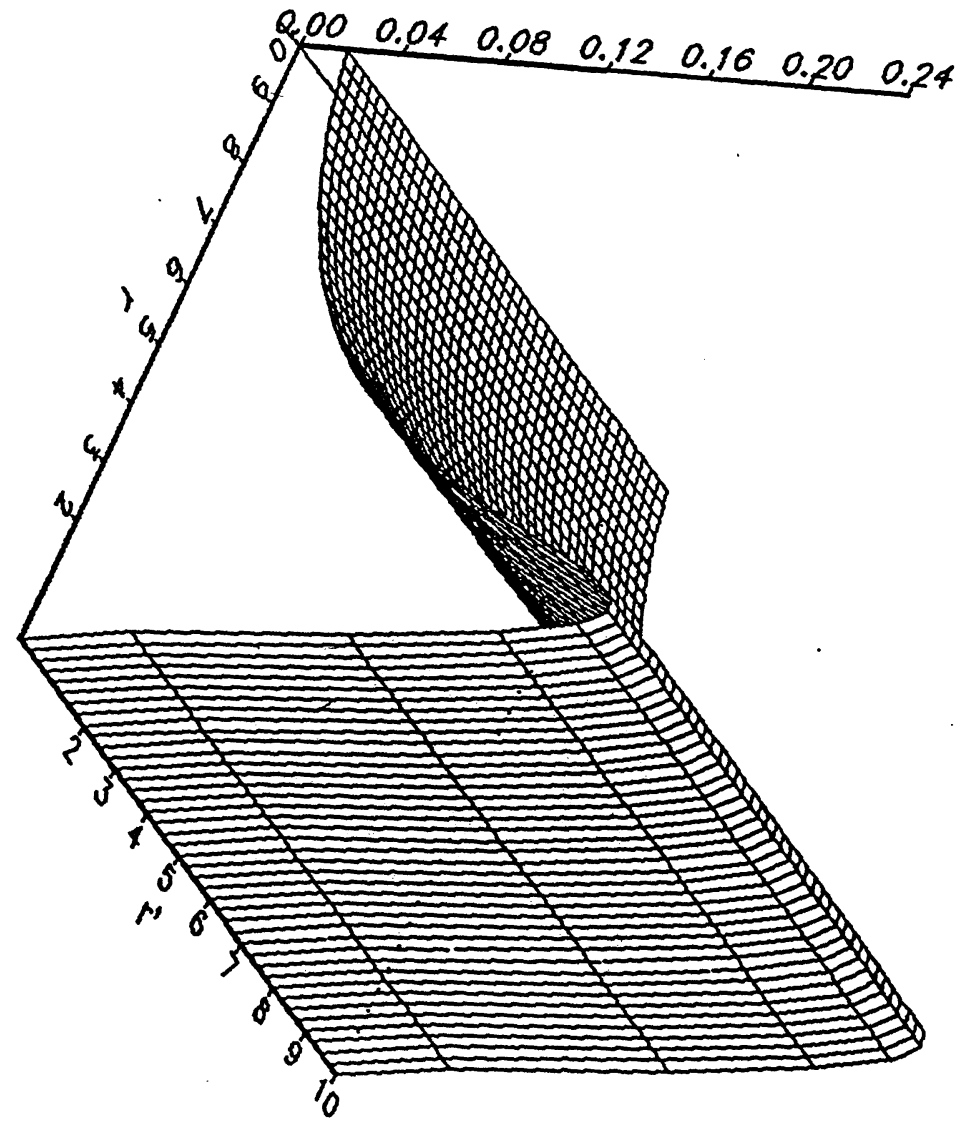




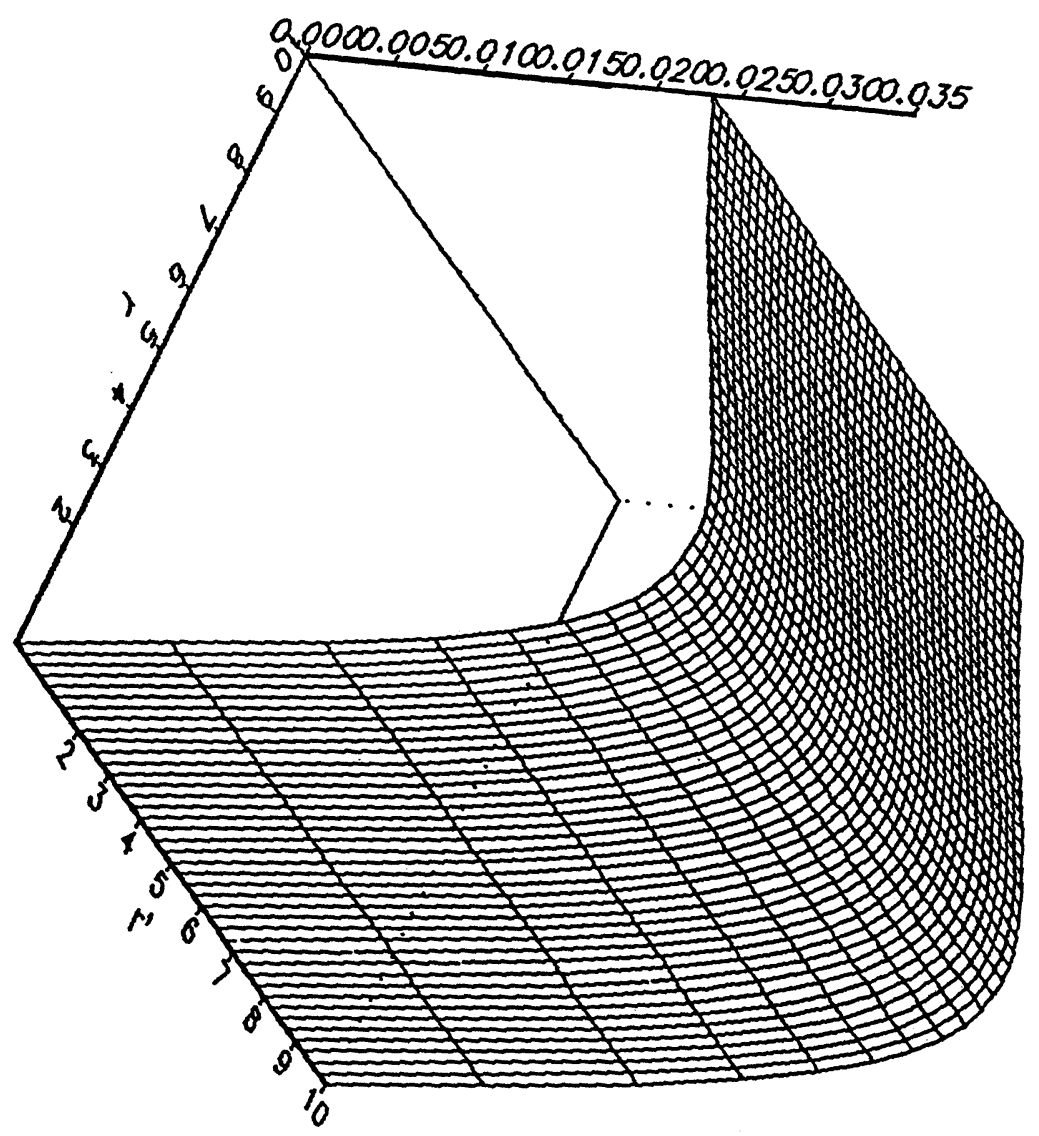

